\title{
Competence in transbronchial cryobiopsy
}

\author{
Emanuela BARISIONE 1 *, Mario SALIO 1, Micaela ROMAGNOLI 2, \\ Alessia PRATICÒ ${ }^{3}$, Elena BARGAGLI ${ }^{4}$, Lorenzo CORBETTA ${ }^{5}$
}

1Unit of Pneumology, San Martino Policlinic Hospital, Genoa, Italy; 2Unit of Interventional Pneumology, Sant'Orsola-Malpighi Policlinic, Bologna, Italy; ${ }^{3}$ Unit of Pneumology, UTIR Azienda Ospedaliera di Perugia, Perugia, Italy; ${ }^{4}$ Unit of Pneumology, University of Siena, Siena, Italy; ${ }^{5}$ Unit of Interventional Pulmonology, University of Florence, Florence, Italy

*Corresponding author: Emanuela Barisione, Unit of Pneumology, San Martino Policlinic Hospital, Genoa, Italy. E-mail: emabarisione@yahoo.it

\section{A B S T R A C T}

Over the last decade transbronchial lung cryobiopsy (TBLC) has proven to be an "innovative application" of an "old procedure" for the histologic diagnosis of diffuse interstitial lung diseases (DILDs). Thus, the technique of TBL cryobiopsy is now adopted for diagnostic purposes, transbronchially in peripheral airways to sample lung parenchyma, whereas this same technique was traditionally employed in the past for therapeutic purposes, essentially for the management of malignant obstruction of central airways. When patients with interstitial lung diseases (ILDs) need histopathological data in their diagnostic pathway, this bioptic approach could be a valid alternative to surgical lung biopsy, that is still the gold standard at the moment. TBL cryobiopsy has a good safety profile, its sensitivity and specificity appear good overall in idiopathic pulmonary fibrosis. In the last ten years, many papers have been published about this procedure defining modalities by which cryobiopsy should be performed. These studies have shown that TBL cryobiopsy is feasible, it allows to obtain larger lung parenchymal specimens ( 3 times larger than "classic" transbronchial biopsies), characterized by unaltered and artefact-free morphology, and it represents a safe and poorly invasive diagnostic tool for the histologic diagnosis of ILDs. The technical aspects are really important, and they still need a complete standardization. TBL cryobiopsy should be part of an equipment of the modern interventional pulmonologist, who should know indications and contraindications of this methodic and the technical aspects of the procedure. This is a complex procedure requiring to be performed by endoscopists working in specialized centers with specific knowledge of DILDs, and a multidisciplinary approach, which represent pre-requisites for admission to training in this procedure.

(Cite this article as: Barisione E, Salio M, Romagnoli M, Praticò A, Bargagli E, Corbetta L. Competence in transbronchial cryobiopsy. Panminerva Med 2019;61:290-7. DOI: 10.23736/S0031-0808.18.03567-X)

KEY WORDS: Bronchoscopy - Pulmonary medicine - Education.

$\mathrm{I}^{\mathrm{n}}$ nterstitial lung diseases (ILD) are a group of lung diseases that can be either of known or unknown cause; according to the statement of the American Thoracic Society and the European Respiratory Society, ILD with known cause include the pneumoconiosis, ILD associated with connective tissue disease (CTD-ILD), and hypersensitivity pneumonitis (HP); ILD with unknown cause are sarcoidosis and idiopathic interstitial pneumonias (IIP). IIP are another heterogenous entity comprising idiopathic pulmonary fibrosis (IPF), nonspecific interstitial pneumonia (NSIP), desquamative interstitial pneumonia (DIP), respiratory bronchiolitis with interstitial lung disease (RBILD), acute interstitial pneumonia (AIP), cryptogenic organizing pneumonia (COP), and lymphoid interstitial pneumonia (LIP).

Idiopathic pulmonary fibrosis (IPF) is the most common of the idiopathic pulmonary fibrotic diseases and has the worst prognosis with a mean life expectancy of 3.8 years. It is defined as a chronic fibrosing interstitial pneumonia of unknown cause with a UIP pattern on high-resolution Computer Tomography (HRCT) scan or on surgical lung biopsy.

The diagnosis of ILD relies on a combination of multiple diagnostic tools, such as imaging technics, blood test, lung function tests, bronchoalveolar lavage (BAL) and lung biopsy.

\section{Knowledge of the diseases and indications}

American Thoracic Society (ATS), European Respiratory Society (ERS), Japanese Respiratory Society (JRS), and 
Latin American Thoracic Society (ALAT) published the revision of the diagnostic recommendations in $2018 .{ }^{2}$

In this document, the new principal recommendations regarding bronchoscopic procedures are the following:

- performing BAL cellular analysis for HRTC pattern of probable UIP, indeterminate and alternative diagnosis, but not for HRTC pattern of UIP.

There was general agreement that BAL is appropriate when the radiologic differential diagnosis includes eosinophilic pneumonia, sarcoidosis, or infection. ${ }^{2}$

In 2009, Oshimo et al. investigated the role of BAL in the diagnosis of known causes of interstitial pneumonia, particularly hypersensitivity pneumonitis. ${ }^{3}$

Some BAL cell type proportions were markedly different in patients with IPF compared with patients with other ILDs. Patients with IPF had a slightly increased proportion of eosinophils compared with healthy individuals but a markedly lower proportion of eosinophils than patients with eosinophilic pneumonia; thus, patients with a markedly elevated proportion of eosinophils are more likely to have eosinophilic pneumonia than IPF. Patients with IPF had a similar to slightly higher proportion of lymphocytes and $\mathrm{CD} 4 / \mathrm{CD} 8$ ratio in their BAL than healthy individuals but a markedly lower proportion of lymphocytes and CD4/CD8 ratio in their BAL than patients with sarcoidosis; thus, patients with a markedly elevated proportion of lymphocytes and CD4/CD8 ratio are more likely to have sarcoidosis than IPF;2

- performing surgical lung biopsy for HRTC pattern of probable UIP, indeterminate and alternative diagnosis, but not for HRTC pattern of UIP.

The surgical lung biopsy could be performed when the desirable consequences (adequate specimens in 100\%, diagnosis made in $89 \%$ ) were weighed against the undesirable consequences (surgical complications including mortality, exacerbations, respiratory infection, bleeding, prolonged air leak). Video-assisted thoracoscopic surgery is the preferred approach to SLB for patients who can tolerate single-lung ventilation, rather than open thoracotomy. ${ }^{2}$ In appropriately selected patients, an SLB provides valuable diagnostic information that cannot be obtained by any other means with low risk of complication. However, an SLB is unnecessary when a confident UIP chest imaging pattern is seen on HRCT scanning in the clinical context of an IIP, nor is it routinely regularly needed in patients with a well-defined CTD-ILD and those with a specific, clinically significant, and temporally appropriate drug, occupational, avocational, or accidental exposure. SLBs are generally unhelpful in patients with acute respiratory fail- ure and a new diagnosis of fibrosing ILD. Severe hypoxia, respiratory failure, immunocompromised status, and rapidly progressive disease prior to biopsy are associated with increased morbidity and mortality. Multidisciplinary discussion, using the available clinical, HRCT, and SLB data, is useful in making a definitive diagnosis; however, diagnostic uncertainty may still occur in a minority of patients with ILD; 4

- no recommendation was made either for or against transbronchial lung biopsy.

Among all TBBX, only one-third yielded a specific diagnosis (i.e., the diagnostic yield). ${ }^{2}$ Tommassetti et al. have already reported the lower predictive value of TBBx for UIP pattern. ${ }^{5}$ The major limitation of this evidence could be that the studies did not stratify patients according to HRCT pattern. It was argued that patients whose HRCT pattern is probable UIP, indeterminate, or an alternative diagnosis are significantly more likely to have an etiology detectable by TBBx (e.g., sarcoidosis) than patients with an HRCT pattern of UIP. Thus, if patients had been stratified according to their HRCT pattern, the diagnostic yield and number of SLBs avoided would probably have been higher among those with an HRCT pattern of probable UIP, indeterminate, or an alternative diagnosis and lower among those with an HRCT pattern of UIP;

- no recommendation was made either for or against transbronchial cryobiopsy.

Pooling studies (unweighted) indicated that lung cryobiopsy obtained an adequate sample in the vast majority of cases. Among the adequate samples, a specific diagnosis was obtained in more than four- fifths of cases, with the remaining deemed unclassifiable. Among lung cryobiopsy procedures, the majority yielded an adequate specimen for a specific diagnosis. Additional complications included exacerbations, mild bleeding (e.g., requiring suction to clear but no other endoscopic procedures), moderate bleeding (e.g., requiring endoscopic procedures like bronchial occlusion-collapse and/or instillation of ice-cold saline), and severe bleeding (e.g., causing hemodynamic or respiratory instability, requiring tamponade or other surgical interventions, transfusions or admission to the intensive care unit). Although the panel was enthusiastic about the desirable consequences of lung cryobiopsy (adequate specimens in $96 \%$, SLB avoided in $80 \%$ ), this was offset by concern about the undesirable consequences (nondiagnostic in $20 \%$, risk of procedural complications), lack of standardized procedure and approach (i.e. how many specimens should be obtained to optimize diagnostic yield while minimizing complications? From which portion of 
the lung should they be obtained? For how long should the probe be cooled?), and the heterogeneous rates of adverse events noted in previous studies. The panel concluded that it is reasonable for experienced centers and experts with a track record of performing the procedure safely to continue performing lung cryobiopsy in patients whose HRCT pattern is probable UIP, indeterminate, or an alternative diagnosis. However, the panel believed very strongly and recommends that such experts work toward developing a standardized procedure that optimizes the balance between diagnostic yield and complications. Those who have not yet begun to perform cryobiopsy should wait until the procedure has been standardized before implementing this into clinical practice; ${ }^{2}$

- multidisciplinary discussion, instead, remains central for decision-making. ${ }^{2}$

\section{Knowledge of instruments}

The procedure of transbronchial lung cryobiopsy (TBLC) refers to the use of an endobronchial flexible cryoprobe to obtain endoscopic samples of peripheral lung tissue. The cryosurgical equipment operates based on the JouleThomson effect, which dictates that a compressed gas released at the high flow rapidly expands and create a very low temperature. The cooling agent is applied under high pressure through the central canal of the probe (Erbokryo CA; ERBE, Tübingen, Germany). Carbon dioxide $\left(\mathrm{CO}_{2}\right)$ or nitric oxide are the cooling agents commonly used. The gas at the tip expands due to the sudden difference in the pressure relative to the atmospheric pressure, resulting in a drop-in temperature at the tip of the probe. The gas more frequently used nowadays (mainly for the presence of specific regulatory rules in the majority of country hampering the use of nitric oxide in endoscopic suites) is $\mathrm{CO}_{2}$, although nitric oxide may achieve lower temperatures. ${ }^{6}$ Before 2009, cryoprobe was mostly used in the palliative treatment of obstructing endobronchial tumors. Thus, the use of a flexible cryoprobe with a flexible bronchoscope was first described in the USA in 1996 by Mathur et al. for cryotherapy purposes, reporting relief of large airways obstruction in $90 \%$ of the cases. ${ }^{7}$ Since then, the experience with endobronchial cryotherapy for the management of malignant tumors and non-malignant endobronchial obstruction has broadened, with several published studies reporting favorable safety, effectiveness and cost profile. ${ }^{8,9}$

The utilization of a cryoprobe not for palliative airways disobstruction, but for lung parenchyma diagnostic aims was first applied in 2009 by Babiak et al., in their pilot study for the diagnosis of ILD. ${ }^{10}$ Forty-one patients with radiologic evidence of ILD at the chest HRCT scan underwent, during a flexible bronchoscopy, transbronchial conventional biopsies and transbronchial cryobiopsies, these last ones allowing to obtain larger biopsy samples of lung parenchyma (mean specimen area $5.82 \mathrm{~mm}$ ), and a definitive diagnosis in a substantial number of cases. ${ }^{11} \mathrm{Af}-$ ter this innovative study, this technique has been applied worldwide for the diagnosis of ILDs, showing a reported diagnostic yield of $70 \%$ to $80 \%$, with a much better safety profile than surgical lung biopsy (SLB), although several systematic reviews and meta-analyses have shown that the conclusions reached by most of the publications have been limited by a not negligible heterogeneity between studies. ${ }^{12}$

The cryosurgical equipment is relatively simple, consisting of two elements: 1) the cooling agent - in the vast majority of reports being carbon dioxide - which is applied under high pressure through the central canal of 2) the cryoprobe (ERBE Elektromedizin GmbH, Tübingen, Germany). The cryoprobe consists of a long flexible insulated catheter with blunt metal tip that can be inserted through the working channel of a flexible bronchoscope. The metal tip cools rapidly to extremely cold temperatures based on the Joule-Thomson effect. As compressed gas is released at a high flow rate into the tip of the probe, it rapidly expands and cools to a temperature of $-79^{\circ} \mathrm{C}$ (using carbon dioxide, $-52{ }^{\circ} \mathrm{C}$ is the real temperature developed in the lung tissue) or $-89^{\circ} \mathrm{C}$ (using nitrous oxide) within few seconds. ${ }^{9-11}$ The gas then escapes down the catheter via an efferent channel and is vented to the atmosphere.

\section{Core basic skills}

The basic skills to perform transbronchial cryobiopsy procedures are:

- active participation within the multidisciplinary team, together with radiologists and pathologists;

- competency in flexible and rigid bronchoscopy;

- correct interpretation of chest HRCT scan and ability to locate the area in which there is the greatest probability of obtaining adequate tissue to formulate a conclusive diagnosis;

- ability to position the blocker balloon, so that patient safety is in first place throughout the procedure;

- competency on the use of fluoroscopy to guide the procedures of transbronchial cryobiopsy;

- ability to perform the procedure, to reach the correct area and to achieve the sampling; 
- management of specimens, both in keeping it attached to the probe during the retraction of the flexible bronchoscope and during the thawing of the sample;

- prevention and management of possible complications (pneumothorax, bleeding, complications related to local anesthesia or sedation).

\section{Procedural description and sedation}

Technically, the basic procedure consists of the following steps: with the patient being intubated (rigid bronchoscope or endotracheal tube) the cryoprobe is advanced through the working channel of the flexible bronchoscope into the peripheral lung, and then gas expansion activated for several seconds ( 3 to 7 seconds, depending on the use of the $1.9-\mathrm{mm}$ probe or of the $2.4-\mathrm{mm}$ probe) causing surrounding parenchyma to rapidly freeze and adhere to the cryoprobe tip. The bronchoscope with the inserted cryoprobe and the attached frozen biopsy is then removed enbloc from the airway, since the cryobiopsy is significantly larger than the working channel of the bronchoscope, and thus cannot be extracted through this channel. ${ }^{10}$ Before performing the biopsies, it is recommended to prophylactically insert a Fogarty balloon (4 or $5 \mathrm{~F}$ ), placed in the lobar bronchus near the biopsy segment, and routinely inflated after sampling, to minimize the consequence of hemorrhage. A rigid suction tube must also be available, to be introduced inside the rigid bronchoscope, if needed. Cryoprobe tip with frozen biopsy is then thawed into saline, to rapidly thaw and release the biopsy from the cryoprobe. Biopsy is then fixed in $4 \%$ formalin and embedded in paraffin, and then stained with hematoxylineosin (H\&E) and periodic-acid Schiff (PAS). If using a rigid bronchoscope without prophylactic balloon placement, a long instrument (bronchoscope) should be used. Resulting biopsies tend to be $7-10 \mathrm{~mm}$ in greatest dimension, significantly larger than traditional forceps biopsies (Figure 1, 2), and notably with lack crush artefact (Figure $3,4,5) \cdot{ }^{11}$

Beside these basic technical aspects, the procedure includes several further essential items, e.g. the correct patient selection, the operating setting and equipment, sedation and ventilation, the distance between activated cryoprobe and pleura (Figure 4, 5), optimal number of cryobiopsies, the management of potential correlated risks during and after the procedure (endobronchial hemorrhage and pneumothorax), the literature showing a broad range of procedural techniques currently in use. ${ }^{11}$

Recently, an Expert Statement from the Cryobiopsy

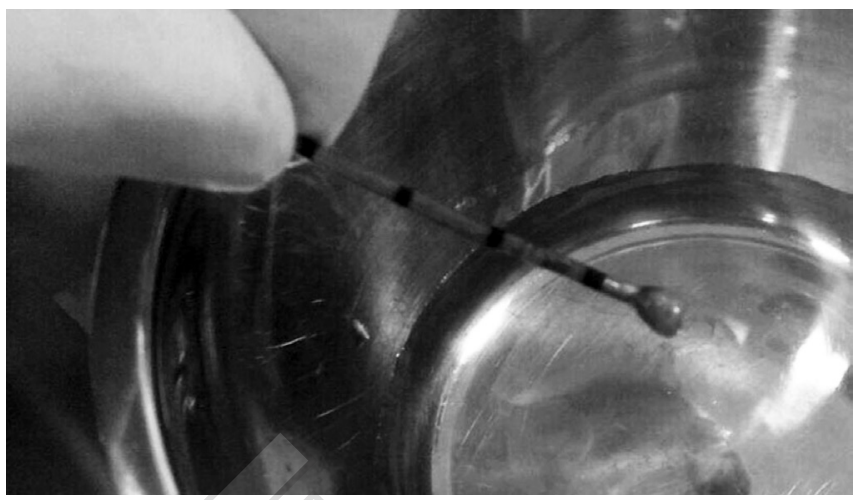

Figure 1.- Tranbronchial cryobiopsy tights to the tip probe.

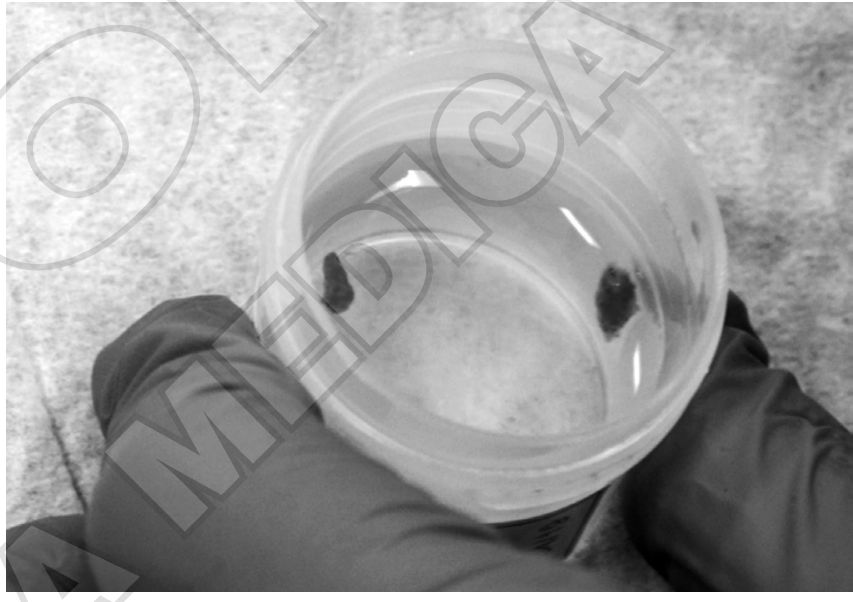

Figure 2.-Transbronchial cryobiopsy in formalin 4\%.

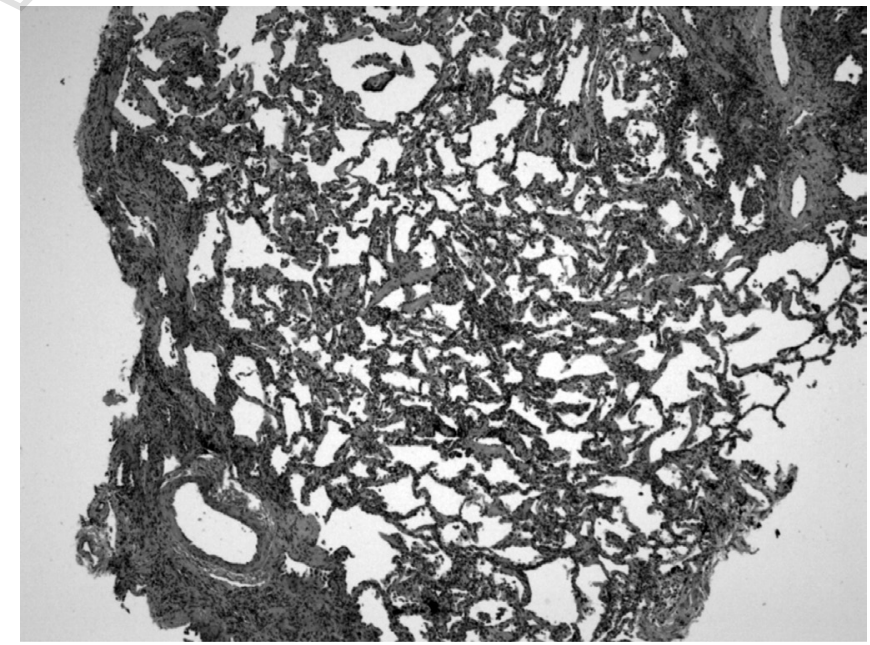

Figure 3.-Transbronchial cryobiopsy specimen with normal lung areas alternating with dense scarring obliterating the alveolar architecture (patchy fibrosis). 


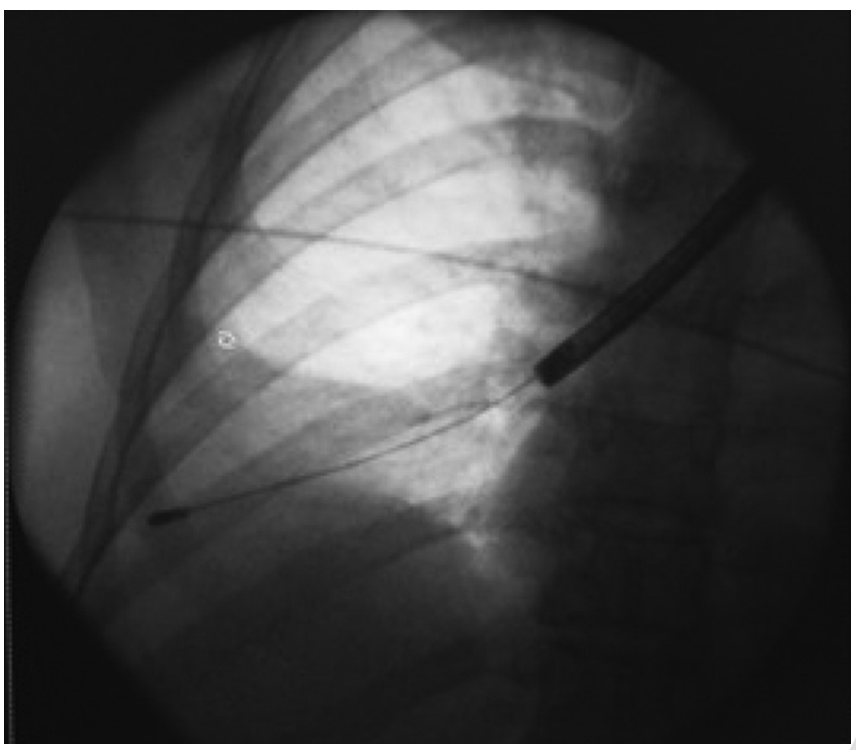

Figure 4.-Fluoroscopic view of the cryoprobe.

Working Group on Safety and Utility and a Call for Standardization of the Procedure has been published, as an attempt to improve the substantial procedural variability among centers, and the lack of a real standardization of this technique: 13

\section{Patient selection}

Patients must be selected for invasive procedures, including TBLC or SLB mainly based on MDA assessment, and so after a multidisciplinary indication for a lung histology need. In general, the indications for TBLC for ILD are the same as for SLB. TBLC (and SLB) are not indicated in subjects showing HRCT images demonstrating a typical UIP pattern, or already having a diagnosis of connective tissue disease, although the Expert Statement suggests that TBLC could sometimes be proposed in these patients, with the aim of obtaining more definitive data suggesting occult exposures or collagen vascular diseases. ${ }^{12}$ Moreover, the same statement suggests that patients with an acute or subacute lung disease which is not rapidly progressing, with a low probability of having acute exacerbation of ILD might benefit from TBLC, ${ }^{13}$ although evidence is scarce, and it is difficult to predict the probability for acute ILD, these cases certainly need to be discussed and carefully evaluated case by case. In general, contraindications for TBLC vary substantially across studies, but it can be suggested that there are no age limits, $\mathrm{FVC}<50 \%$ and $\mathrm{DLCO}<35 \%$ of the predicted values are regarded as

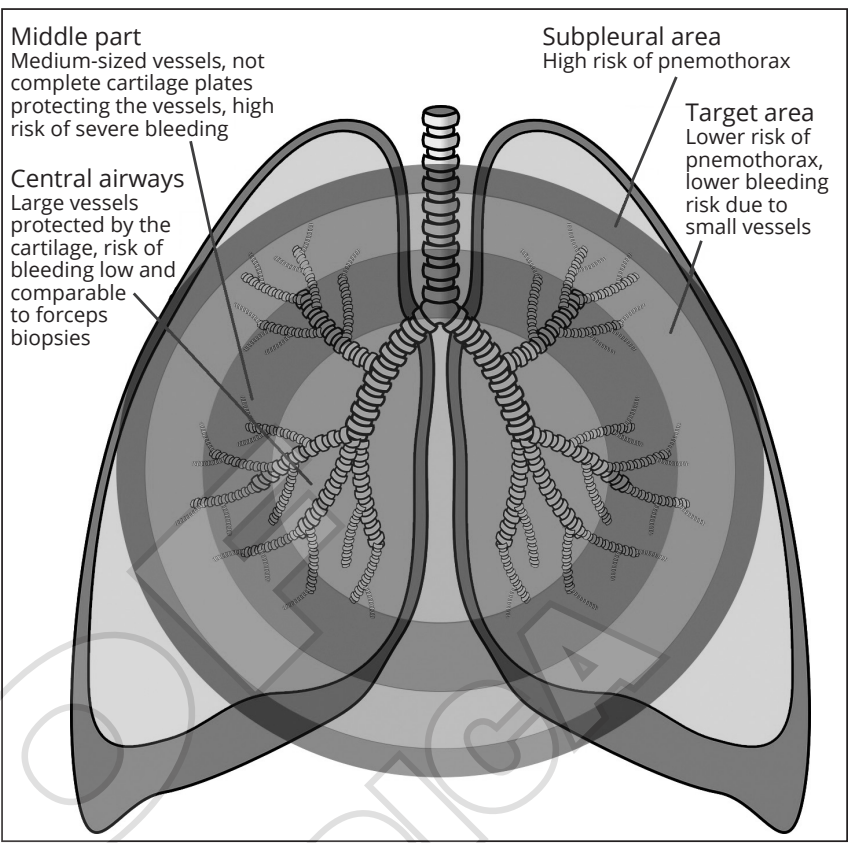

Figure 5.-The biopsies obtained in the periphery of secondary pulmonary lobule are more likely to get appropriate specimens for the histologic diagnosis of UIP. The area to perform the cryobiopsy with lower risk of pneumothorax or bleeding is the Target area. The major risk of pneumothorax is inside the Subpleural area, while the risk of severe bleeding increases if the biopsies are obtained in the middle third of lung.

relative contraindications, and pulmonary hypertension may increase the bleeding risk and is therefore considered as a relative contraindication. ${ }^{13}$

\section{Operating setting and equipment}

TBLC is mainly performed with in-patient setting, but also out-patient setting is conducted among centers. Before starting of the procedure, the operator shall choose the equipment, and check that every item is properly functioning and correctly prepared for the procedure, including all related devices, invasive and non-invasive ventilation support. In particular, cryoprobe must be tested within saline before starting the examination, to check its correct functioning. Prior to the procedure, the most affected segment have been determined on the basis of imaging studies (HRCT). This is the segment to sample. Ideally, two different lobes on the same side, with different segmental areas should be sampled. ${ }^{13}$

There is a substantial heterogeneity in the methodology used across different centers for the performance of TBLC. Airways intubation is highly suggested and it is used by the majority of centers, either with rigid bronchoscope or 
with endotracheal tube, ${ }^{13}$ although some centers perform TBLC only with a flexible bronchoscope through laryngeal mask. ${ }^{14-16}$ The use of prophylactic bronchial blocker placement to control bleeding is highly suggested, although some authors performed TBLC without the positioning of any bronchial blocker. ${ }^{17}$ In fact, before performing cryobiopsies, it is recommended to prophylactically insert a Fogarty balloon (4 or $5 \mathrm{~F}$ ) or a bronchial blocker, placed in the lobar bronchus near the biopsy segment, and routinely inflated after sampling, to minimize the consequence of hemorrhage. A rigid suction tube should also be available, to introduce it inside the rigid bronchoscope, if needed.

The use of a fluoroscopic guidance during the procedure is mandatory, to control in real-time the right positioning of the cryoprobe into the lung parenchyma, and the right distance from the pleura at the time of the cooling.

\section{Sedation and ventilation}

When performing TBLC, general anesthesia or deep sedation and intubation are recommended. ${ }^{13}$ Most of the centers use intubation with deep sedation. ${ }^{18}$ However, in some centers the procedure is conducted during conscious sedation, which does not seem to interfere with the final success of the procedure. ${ }^{19-21}$ Jet ventilation has also been used for TBLC in ILD, with similar incidence of pneumothorax $(19 \%)$, although bleeding seemed to represent an issue in this report which showed moderate endobronchial bleeding in $25 \%$ of patients, and severe bleeding in a high percentage $(53 \%)$ of cases. 22

\section{Distance between cryoprobe and pleura}

Once the flexible cryoprobe is introduced through the working channel of the bronchoscope under fluoroscopic guidance, it should ideally reach a sub-segmental bronchus that allows the probe to be perpendicular to the chest wall: this allows the operator to ensure that the distance from the wall stays at $1 \mathrm{~cm}$, in order to avoid a too peripheral positioning of the cryoprobe (potentially causing pneumothorax) or a too central positioning (with a risk for bleeding).

\section{The optimal number and sites of cryobiopsies}

The optimal number of cryobiopsies cannot be uniformly established, although in the majority of centers 3-5 biopsies are usually taken. ${ }^{13}$ The quantity of samples needed for diagnosis is influenced by the quality of the specimen, the heterogeneity and the pattern of the disease. A recent study suggested that there is higher diagnostic yield when biopsies are sampled from different segments within the same lobe, compared to biopsies in the same segment. ${ }^{23}$ Ideally, similarly to SLB technique, TBLC should also be taken from 2 different lobes, but no data are available yet to demonstrate it.

\section{The management of potential correlated risks}

Endobronchial hemorrhage and pneumothorax represent the two most frequent complications after TBLC. Intubation either with the rigid bronchoscope or a flexible endotracheal tube is highly recommended to maintain an access to the airways in case of a severe bleeding after a TBLC. As already mentioned, the inflation of a Fogarty balloon or a bronchial blocker (placed in the bronchus leading to the biopsied area) immediately after TBLC prevents central airways in case of significant bleeding.

Concerning the assessment of the occurrence of pneumothorax, a postprocedural chest X-ray should be performed, either immediately in case of symptoms, or $2 \mathrm{~h}$ after the end of the procedure if the patient is asymptomatic, especially in case of an out-patient setting.

\section{Other potential applications of cryoprobe}

Rigid cryoprobe has been used in the airways since 1968 in the palliative treatment of obstructing endobronchial tumors. 8

Flexible cryoprobe can be used to perform biopsies on endobronchial lesions.

A prospective, randomized multicenter study ${ }^{24}$ has evaluated the diagnostic yield of cryobiopsies in comparison with forceps biopsies in 563 patients with a final diagnosis of cancer. A definitive diagnosis was achieved in $85.1 \%$ of patients randomized to conventional forceps biopsy and $95.0 \%$ of patients who underwent cryobiopsy $(\mathrm{P}<0.001)$. There was no difference in the incidence of significant bleeding.

The flexible cryoprobe can also be useful for extracting foreign bodies from the respiratory tract. ${ }^{25,} 26$ The cryoprobe can be used with a flexible technique or in combination with a rigid bronchoscope. The possibility of extracting a foreign body depends on its composition and in particular its water content. The use of cryoprobe can reduce the risk of foreign-body dislocation.

Some studies evaluated the feasibility, safety and diagnostic value of using a flexible cryoprobe to obtain parietal pleural biopsies during pleuroscopy. The efficacy of pleural cryobiopsy was compared only with the flexible forceps and not with the rigid forceps which represents the standard in pleuroscopy. ${ }^{27}$ 


\section{Theoretical and practical training}

A practical training should include: 28

- video and other multimedia resource;

- live session and case report;

- task trainer simulation and non-technical skill simulation for training on how to manage complications within the multidisciplinary team, including anesthesiologist and nurse;

- training on animal models and cadavers;

- supervised training on patients.

\section{Quantitative and qualitative assessment}

Currently, in literature, there is not a minimum of procedures to be considered expert operators nor a minimum of one-year procedures to maintain the competence. 28

Trainee should be in attendance on at least 10 procedures performed by expert bronchoscopists (ACCP). Trainee should perform directly, as first operator, at least 10 procedures under supervision of an expert bronchoscopist. Trainee should be in attendance on a session with a major complication of bleeding and tension pneumothorax.

The competence maintenance should be 5-10 procedures/year.

For qualitative assessment, MCQ core-based questionnaires and creation of a specific STAT would be useful.

The outcome measures are the following:

- percentage (and number) of biopsies taken for every procedure;

- percentage of complications, in all procedures;

- outcome of complications.

\section{References}

1. Travis WD, Costabel U, Hansell DM, King TE Jr, Lynch DA, Nicholson AG, et al.; ATS/ERS Committee on Idiopathic Interstitial Pneumonias. An official American Thoracic Society/European Respiratory Society statement: update of the international multidisciplinary classification of the idiopathic interstitial pneumonias. Am J Respir Crit Care Med 2013;188:733-48.

2. Raghu G, Remy-Jardin M, Myers JL, Richeldi L, Ryerson CJ, Lederer DJ, et al.; American Thoracic Society, European Respiratory Society, Japanese Respiratory Society, and Latin American Thoracic Society. Diagnosis of Idiopathic Pulmonary Fibrosis. An Official ATS/ERS/ JRS/ALAT Clinical Practice Guideline. Am J Respir Crit Care Med 2018;198:e44-68.

3. Ohshimo S, Bonella F, Cui A, Beume M, Kohno N, Guzman J, et al. Significance of bronchoalveolar lavage for the diagnosis of idiopathic pulmonary fibrosis. Am J Respir Crit Care Med 2009;179:1043-7.

4. Raj R, Raparia K, Lynch DA, Brown KK. Surgical Lung Biopsy for Interstitial Lung Diseases. Chest 2017;151:1131-40.
5. Tomassetti S, Cavazza A, Colby TV, Ryu JH, Nanni O, Scarpi E, et al. Transbronchial biopsy is useful in predicting UIP pattern. Respir Res 2012;13:96

6. Poletti V, Hetzel J. Transbronchial Cryobiopsy in Diffuse Parenchymal Lung Disease: Need for Procedural Standardization. Respiration 2015;90:275-8

7. Mathur PN, Wolf KM, Busk MF, Briete WM, Datzman M. Fiberoptic bronchoscopic cryotherapy in the management of tracheobronchial obstruction. Chest 1996;110:718-23.

8. Hetzel M, Hetzel J, Schumann C, Marx N, Babiak A. Cryorecanalization: a new approach for the immediate management of acute airway obstruction. J Thorac Cardiovasc Surg 2004;127:1427-31.

9. Reddy AJ, Govert JA, Sporn TA, Wahidi MM. Broncholith removal using cryotherapy during flexible bronchoscopy: a case report. Chest 2007;132:1661-3.

10. Babiak A, Hetzel J, Krishna G, Fritz P, Moeller P, Balli T, et al. Transbronchial cryobiopsy: a new tool for lung biopsies. Respiration 2009;78:203-8

11. Lentz RJ, Argento AC, Colby TV, Rickman OB, Maldonado F. Transbronchial cryobiopsy for diffuse parenchymal lung disease: a state-of-theart review of procedural techniques, current evidence, and future challenges. J Thorac Dis 2017;9:2186-203.

12. Casoni GL, Tomassetti S, Cavazza A, Colby TV, Dubini A, Ryu JH, et al. Transbronchial lung cryobiopsy in the diagnosis of fibrotic interstitial lung diseases. PLoS One 2014;9:e86716.

13. Hetzel J, Maldonado F, Ravaglia C, Wells AU, Colby TV, Tomassetti $\mathrm{S}$, et al. Transbronchial Cryobiopsies for the Diagnosis of Diffuse Parenchymal Lung Diseases: Expert Statement from the Cryobiopsy Working Group on Safety and Utility and a Call for Standardization of the Procedure. Respiration 2018;95:188-200.

14. Sastre JA, Cordovilla R, Jiménez MF, López T. Management of a

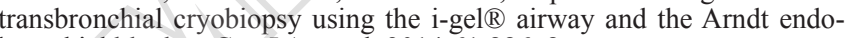
bronchial blocker. Can J Anaesth 2014;61:886-8.

15. Schmutz A, Dürk T, Idzko M, Koehler T, Kalbhenn J, Loop T. Feasibility of a Supraglottic Airway Device for Transbronchial Lung Cryobiopsy-A Retrospective Analysis. J Cardiothorac Vasc Anesth 2017;31:1343-7.

16. Gelzinis TA. Supraglottic airway device for transbronchial lung cryobiopsy. J Cardiothorac Vasc Anesth 2017;31:1348-50.

17. Gershman E, Fruchter O, Benjamin F, Nader AR, Rosengarten D, Rusanov V, et al. Safety of cryo-transbronchial biopsy in diffuse lung diseases: analysis of three hundred cases. Respiration 2015;90:40-6.

18. Ravaglia C, Bonifazi M, Wells AU, Tomassetti S, Gurioli C, Piciucchi S, et al. Safety and Diagnostic Yield of Transbronchial Lung Cryobiopsy in Diffuse Parenchymal Lung Diseases: A Comparative Study versus Video-Assisted Thoracoscopic Lung Biopsy and a Systematic Review of the Literature. Respiration 2016;91:215-27.

19. Griff S, Schönfeld N, Ammenwerth W, Blum TG, Grah C, Bauer TT, et al. Diagnostic yield of transbronchial cryobiopsy in non-neoplastic lung disease: a retrospective case series. BMC Pulm Med 2014;14:171.

20. Fruchter O, Fridel L, Rosengarten D, Rahman NA, Kramer MR. Transbronchial cryobiopsy in immunocompromised patients with pulmonary infiltrates: a pilot study. Hai 2013;91:619-24.

21. Fruchter O, Fridel L, El Raouf BA, Abdel-Rahman N, Rosengarten D, Kramer MR. Histological diagnosis of interstitial lung diseases by cryotransbronchial biopsy. Respirology 2014;19:683-8.

22. Hagmeyer L, Theegarten D, Wohlschläger J, Treml M, Matthes S, Priegnitz C, et al. The role of transbronchial cryobiopsy and surgical lung biopsy in the diagnostic algorithm of interstitial lung disease. Clin Respir J 2016;10:589-95.

23. Ravaglia C, Wells AU, Tomassetti S, Dubini A, Cavazza A, Piciucchi S, et al. Transbronchial Lung Cryobiopsy in Diffuse Parenchymal Lung Disease: Comparison between Biopsy from 1 Segment and Biop- 
sy from 2 Segments - Diagnostic Yield and Complications. Respiration 2017;93:285-92.

24. Hetzel J, Eberhardt R, Herth FJ, Petermann C, Reichle G, Freitag L, et al. Cryobiopsy increases the diagnostic yield of endobronchial biopsy: a multicentre trial. Eur Respir J 2012;39:685-90.

25. Schumann C, Kropf C, Rüdiger S, Wibmer T, Stoiber KM, Lepper PM. Removal of an aspirated foreign body with a flexible cryoprobe. Respir Care 2010;55:1097-9.
26. Sehgal IS, Dhooria S, Agarwal R, Behera D. Use of a flexible cryoprobe for removal of tracheobronchial blood clots. Respir Care 2015;60:e128-31.

27. Maturu VN, Sehgal IS, Dhooria S, Bal A, Aggarwal AN, Behera D, et al. Pleuroscopic cryobiopsy: case series and systematic review. J Bronchology Interv Pulmonol 2015;22:e11-3.

28. Corbetta L, Patelli M. Executive summary of training and competence standards for the interventional pulmonology master program in Italy. J Bronchology Interv Pulmonol 2018;1:6-8.

Conflicts of interest.-The authors certify that there is no conflict of interest with any financial organization regarding the material discussed in the manuscript. Article first published online: October 31, 2018. - Manuscript accepted: October 16, 2018. - Manuscript received: October 15, 2018.

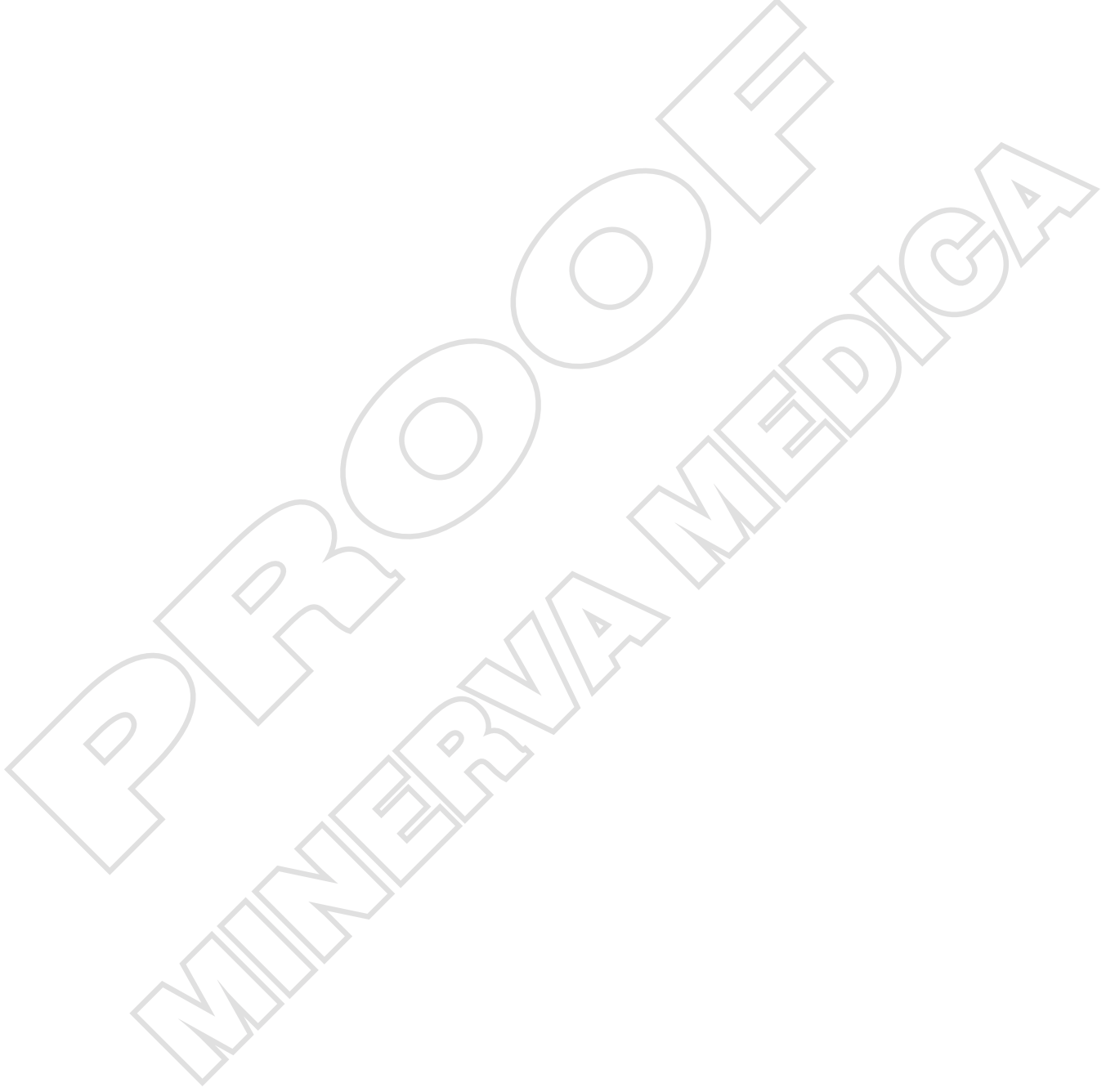

